[0212-7199 (2003) 20: 4; pp 183-186] ANALES DE MEDICINA INTERNA Copyright @ 2003 ARAN EDICIONES, S.L.

AN. MED. INTERNA (Madrid) Vol. 20, N. ${ }^{\circ} 4$, pp. 183-186, 2003

\section{Sintomatología respiratoria y función pulmonar en población geriátrica de una comunidad rural gallega: un estudio piloto}

\author{
F. J. GONZÁLEZ BARCALA, B. TAKKOUCHE", A. MONTES MARTÍNEZ1, \\ C. ZAMARRÓN SANZ, M. SALGUEIRO RODRÍGUEZ, J. R. RODRÍGUEZ SUÁREZ
}

Servicio de Neumología. Hospital Clínico Universitario. ${ }^{1}$ Departamento de Medicina Preventiva. Universidad de Santiago de Compostela
RESPIRATORY SYMPTOMS AND LUNG FUNCTION IN A GERIATRIC POPULATION OF A GALICIAN RURAL COMMUNITY: A PILOT STUDY

\section{RESUMEN}

Introducción: Las enfermedades respiratorias representan una causa frecuente de demanda sanitaria, con significativa repercusión en la morbimortalidad de la población de nuestra comunidad, en especial en la población geriátrica. Resultados de trabajos recientes muestran que el manejo diagnóstico y terapéutico de estos procesos no parece adecuado, relacionándose ésto con el incremento del consumo de recursos sanitarios.

Material y métodos: Se han estudiado 28 individuos del municipio de Valle del Dubra, entre 65 y 74 años. Se ha realizado una exploración espirométrica y una entrevista por cuestionario sobre síntomas respiratorios, estilo de vida, antecedentes personales sanitarios y laborales.

Resultados: El 54\% de los varones de esta población son o han sido fumadores, mientras nunca ha fumado ninguna de las mujeres. Los síntomas respiratorios son más frecuentes entre el sexo femenino, refiriendo algún síntoma el $80 \%$ de estas y el $54 \%$ de los varones. En la espirometría los mayores volúmenes y flujos se obtienen en el sexo masculino, entre los no fumadores y entre los que no refieren disnea.

Discusión: La sintomatología respiratoria parece ser frecuente entre la población rural gallega entre 65 y 74 años. La prevalencia de tabaquismo es similar a otras comunidades de nuestro país, observándose un comportamiento distinto en poblaciones de otros países. El sexo masculino, la condición de no fumador y la ausencia de síntomas respiratorios parecen asociarse con mayores valores espirométricos.

PALABRAS CLAVE: Síntomas respiratorios. Función pulmonar. Población geriátrica.

\section{ABSTRACT}

Introduction: Respiratory diseases are a frequent cause of health demands and have a large impact on morbidity and mortality of the Galician population, especially among the older one. Recent work shows that the diagnosis and treatment of these diseases is not optimal. This increases the utilisation of health care ressources.

Material and methods: We studied 28 patients of the municipality of Val del Dubra (Northwest Spain) aged between 65 and 74 years. We performed a spirometric exploration and carried out a questionnaire interview on respiratory symptoms, life style, and occupational and healthrelated antecedents.

Results: Among men, 54\% of were or are smokers. None of the women ever smoked. Respiratory symptoms were more frequent among women than among men ( $80 \%$ versus $54 \%$ ). In the spirometric study, the largest volumes and flux are observed among non-smoking males who do not report dyspnea.

Discussion: Respiratory symptoms are frequent in the rural population aged between 65 and 74 years. Tobacco consumption is similar to other Spanish communities, but different from that seen in other countries. Male gender, non-smoking status and absence of respiratory symptoms are associated with higher spirometric figures.

KEY WORDS: Respiratory symptoms. Lung function. Geriatric population.

González Barcala FJ, Takkouche B, Montes Martínez, A, Zamarrón Sanz C, Salgueiro Rodríguez, M, Rodríguez. Suárez JR. Sintomatología respiratoria y función pulmonar en población geriátrica de una comunidad rural gallega: un estudio piloto. An Med Interna (Madrid) 2003; 20: $183-186$.

\section{INTRODUCCIÓN}

Los síntomas respiratorios representan una de las causas más frecuentes de requirimiento de asistencia sanitaria por los ciudadanos. La tos es la segunda causa de consulta en atención primaria (1), y el $48 \%$ de la población española refiere algún síntoma respiratorio (2). En cuanto a la población geriá- trica, la presencia de antecedentes respiratorios se asocia con formas de presentación típica de las neumonías (3), siendo además la disnea, tos y expectoración tres de los cuatro síntomas más frecuentes en la neumonía bacteriémica adquirida en la comunidad (4). Asimismo, los cuestionarios de síntomas parecen útiles para orientar el diagnóstico en este grupo de enfermedades (5). 
En Galicia suponen la tercera causa de mortalidad, después de las enfermedades del aparato cardiocirculatorio y los tumores (6).

Se observa, además, tendencia a aumentar de estas patologías en todos los grupos de población, pero fundamentalmente entre mujeres y ancianos (7).

Por otra parte, es conocida la utilidad de la espirometría en el diagnóstico y seguimiento de las enfemedades respiratorias (8-9), asociándose su correcta utilización a un mejor manejo farmacológico de estas patologías, menor frecuencia de agudizaciones, menor consumo de recursos sanitarios y menor morbilidad $(10,11)$. Sin embargo, solo en el $20 \%$ de los hospitales españoles se cumplen las normativas de las sociedades científicas en la realización de esta exploración (12), y a más de la mitad de los pacientes con patología obstructiva nunca se les ha realizado una espirometría (11).

En cuanto a la atención primaria, trabajos recientes han demostrado que menos del $30 \%$ de los médicos de este nivel conocen y siguen las normativas nacionales e internacionales sobre el tratamiento de las infecciones de las vías respiratorias bajas, y que las pautas terapéuticas utilizadas, tanto en la EPOC estable como agudizada, diferían en bastantes aspectos de lo aconsejado por los comités de expertos (11). Además el proyecto PADOC, ha demostrado la utilidad del cribado de la EPOC en atención primaria, permitiendo detectar hasta un $22 \%$ de posibles casos (13).

\section{MATERIAL Y MÉTODOS}

Han sido estudiados 28 individuos residentes en el Valle del Dubra, municipio eminentemente agrícola, empadronados en el municipio y con residencia habitual en el mismo. Estos sujetos no están institucionalizados, son de edades comprendidas entre 65 y 74 años, ambos inclusive, y en ellos no se observaron contraindicaciones para la realización de las exploraciones. Fueron capaces de colaborar correctamente y no refirieron agudizaciones en los últimos tres meses.

Los pacientes fueron citados telefónicamente, a través de su médico de familia, en grupos de 10, en el centro de salud de referencia.

Todas las exploraciones fueron realizadas por dos médicos, teniendo uno de ellos experiencia en exploración funcional respiratoria, de acuerdo con los protocolos establecidos por las sociedades científicas, incluyendo una prueba broncodilatadora con salbutamol (9).

Previamente a la realización de las exploraciones, los participantes en el estudio respondieron a un cuestionario sobre estilo de vida, antecedentes personales sanitarios, actividad laboral realizada, síntomas respiratorios, medicación a la que son sometidos y evaluación de la disnea según la escala del British Medical Research Council (14).

Fue motivo de exclusión la colaboración inadecuada en la realización de la maniobra espirométrica o incapacidad para la realización de la misma, no responder a los cuestionarios o responder de forma incorrecta, rechazar la inclusión en el estudio, antecedente de tratamiento las horas previas a realizar la espirometría con fármacos con actividad conocida sobre la función respiratoria, presencia de enfermedad respiratoria aguda en el momento de la exploración, rinitis en los tres días previos a la exploración, infecciones de la vía aérea superior en las tres últimas semanas previas a la exploración, y presentación de alguno de los criterios de contraindicación para la realización de exploración funcional respiratoria.

Las preguntas del cuestionario son elaboradas a partir del cuestionario breve de síntomas respiratorios de la European Community Respiratory Health Survey, ya utilizado en nuestro país (15), y del cuestionario de síntomas bronquiales de la International Union Against Tuberculosis and Lung Disease $(5,16)$.

\section{RESULTADOS}

Han sido estudiados 28 individuos, 13 varones y 15 mujeres, cuyas características antropométricas, función pulmonar,

TABLA I

FUNCIÓN PULM ONAR Y ANTRO PO METRÍA (M EDIA $\pm D E)$

\begin{tabular}{lcc}
\hline & Varones & M ujeres \\
$\mathrm{N}$ & 13 & 15 \\
\hline Edad (años) & $68,00 \pm 2,42$ & $69,47 \pm 3,04$ \\
FVC (mililitros) & $3.377 \pm 672$ & $2.707 \pm 440$ \\
FEV1 (mililitros) & $2.630 \pm 533$ & $2.068 \pm 454$ \\
FEV 1/FVC (\%) & $78,00 \pm 7,30$ & $75,93 \pm 8,45$ \\
PEF (mililitros/ segundo) & $6.392 \pm 1372$ & $4.932 \pm 1.143$ \\
FEF25-75 (mililitros/ & & \\
$\quad$ segundo) & $2.656+922$ & $2.227 \pm 1.129$ \\
Talla (centímetros) & $162,08 \pm 5,22$ & $149,60 \pm 6,58$ \\
Peso (kilogramos) & $82,77 \pm 7,51$ & $69,40 \pm 13,77$ \\
IM C (kilogramos/metro & & \\
$\quad$ cuadrado) & $31,50 \pm 2,53$ & $30,80 \pm 4,62$ \\
Superficie corporal & $1,87 \pm 0,10$ & $1,63 \pm 0,18$ \\
\hline
\end{tabular}

hábito tabáquico, actividad física habitual y síntomas respiratorios, incluida disnea, presentamos en las tablas I y II.

El tabaquismo en nuestra población está limitado al sexo masculino, suponiendo los fumadores activos y los exfumadores el 54\% del total de los varones estudiados, continuando en el momento actual con dicho hábito el $23 \%$ de dicha población. Entre las mujeres, no fuma ninguna de las que respondieron a la encuesta de hábito tabáquico. La exposición pasiva al humo del tabaco sólo la refieren dos mujeres y ninguno de los varones (Tabla II) .

La disnea y los síntomas respiratorios son más frecuentes en el sexo femenino. Más del $50 \%$ de los varones y el $80 \%$ de las mujeres presentan algún síntoma respiratorio (Tabla II). Los síntomas más frecuentemente referidos son en los varones pitos o silbidos, pitos durante los episodios de resfriados, dificultad para respirar tras ejercicio y tos por las mañanas. En las mujeres son despertar por ataque de tos, falta de aire con polvo o animales, pitos o silbidos, tos por las mañanas, falta de aire con humos u olores fuertes (Tabla II).

En la exploración espirométrica, los varones presentan mayores volúmenes y flujos que las mujeres (Tabla I). Asimismo se observan valores espirométricos superiores entre los 
TABLA II

\begin{tabular}{|c|c|c|}
\hline \multicolumn{3}{|c|}{ SÍNTO M AS RESPIRATO RIOS Y HÁBITO TABÁQUICO } \\
\hline $\mathrm{n}$ & $\begin{array}{c}\text { Varones } \\
13\end{array}$ & $\begin{array}{c}\text { M ujeres } \\
15\end{array}$ \\
\hline Fumador & $3(23,1 \%)$ & 0 \\
\hline No fumador & $6(46,2 \%)$ & 15 \\
\hline Exfumador & $4(30,8 \%)$ & 0 \\
\hline Fumador pasivo & 0 & 2 \\
\hline Horas de paseo/día (media \pm de) 7 & $7,46 \pm 3,60$ & $8,07 \pm 2,97$ \\
\hline Horas de cama/ día & $8,31 \pm 1,25$ & $9,27 \pm 1,28$ \\
\hline Horas de tv/ día & $1,73 \pm 1,49$ & $1,73 \pm 1,03$ \\
\hline Disnea & 2 & 8 \\
\hline Algún síntoma & 7 & 12 \\
\hline Ningún síntoma & 6 & 3 \\
\hline Pitos o silbidos & 4 & 7 \\
\hline \multicolumn{3}{|l|}{ O presión de pecho por la } \\
\hline $\begin{array}{l}\text { noche } 0 \text { al levantarse } \\
\text { Falta de aire en presencia }\end{array}$ & 0 & 3 \\
\hline de silbidos & 2 & 5 \\
\hline Pitos durante resfriados & 3 & 4 \\
\hline \multicolumn{3}{|l|}{ Dificultad repentina para } \\
\hline respirar sin esfuerzo & 0 & 1 \\
\hline Dificultad para respirar tras ejercicio & 3 & 5 \\
\hline Despertar con dificultad para respira & $\operatorname{rar} 1$ & 1 \\
\hline Despertar por ataque de tos & 1 & 10 \\
\hline Tos por las mañanas & 3 & 7 \\
\hline Flemas al levantarse & 1 & 6 \\
\hline Flemas matutinas más de 3 meses & 0 & 5 \\
\hline \multicolumn{3}{|l|}{ O presión en el pecho con polvo o } \\
\hline animales & 1 & 3 \\
\hline $\begin{array}{l}\text { Falta de aire con polvo } 0 \text { animales } \\
\text { O presión en el pecho con humo }\end{array}$ & 2 & 9 \\
\hline u olores fuertes & 1 & 4 \\
\hline \multicolumn{3}{|l|}{ Falta de aire con humo u olores } \\
\hline fuertes & 2 & 7 \\
\hline Ha tenido asma & 0 & 1 \\
\hline Ha tenido algún ataque de asma & 0 & 0 \\
\hline
\end{tabular}

no fumadores comparados con los fumadores; así como en los que no presentan disnea cuando se comparan con los que refieren cualquier grado de la misma (datos no mostrados). Esta tendencia no se observa ante la presencia o ausencia de otros síntomas respiratorios (datos no mostrados).

\section{DISCUSIÓN}

La prevalencia de sintomatología respiratoria es elevada en este municipio, y al contrario de lo observado en la literatura habitualmente, esta es más frecuente en el sexo femenino, presentando más del $50 \%$ de los varones y el $80 \%$ de las mujeres algún síntoma. Estas cifras son superiores a las obtenidas en otros estudios. En el estudio IBERPOC, abarcando población de cinco áreas españolas entre 40 y 69 años, refieren algún síntoma el $48 \%$ de la población, siendo estos más frecuentes en el sexo masculino, al contrario de lo que sucede en nuestro estudio (2). En otro estudio con población rural catalana la sintomatología referida se aproxima más a la nuestra, con una prevalencia del $65 \%$, pero de predominio en los varones (17). En el estudio de Sevilla, con población más joven, presentaban algún tipo de síntoma el $49 \%$ de la población, con predilección por el sexo masculino (15). También en el estudio de Valencia la prevalencia de síntomas es mayor en el sexo masculino (18).

En esta población no fuma ninguna de las mujeres. En otros estudios de población española, la proporción de fumadoras no supera el $13 \%$, ni el $5 \%$ la de exfumadoras (17-20). Entre los varones predominan los fumadores, con un $23 \%$ de fumadores activos, un $30 \%$ de exfumadores y un $46 \%$ de nunca fumadores. La proporción de expuestos al tabaco es similar a la reseñada por otros trabajos realizados en nuestro país, aunque con mayor porcentaje de fumadores que de exfumadores, en probable relación con la mayor edad de nuestra población $(17,19,20)$. El estudio de Brotóns refleja, en población de menor edad, mayor exposición al tabaquismo, con un $53 \%$ de fumadores activos y un $25 \%$ de exfumadores (18).

En el CHS, en un rango de edad similar al nuestro, hay menos fumadores activos entre los varones, $12 \%$, y más nunca fumadores, $56 \%$. Por el contrario fuman un $11 \%$ de mujeres, y otro $57 \%$ ha fumado (21).

En cuanto a la espirometría, como se observa en otras poblaciones, en el sexo masculino se obtienen valores superiores a los del sexo femenino en todos los parámetros espirométricos estudiados. Diversos autores han analizado esta circunstancia relacionándola con distintos factores como la diferencia en fuerza muscular (22-27), distintos tamaños de tronco y pulmones (28-34), diferencias en el número de alvéolos que son más numerosos en los varones (33), y diferencias en la actividad física (35).

La población estudiada en el HHP, de mayor edad, presenta valores de FP inferiores a los nuestros (36). En relación con los pacientes del CHS, también de mayor edad, la FVC de los varones es superior a la de nuestra población, siendo inferior el FEV1, así como ambos parámetros en las mujeres; siendo destacable el elevado porcentaje de fumadoras y exfumadoras de este grupo poblacional (21). Otros autores obtienen parámetros espirométricos, en general, superiores; pero la edad de nuestra población es superior a la de los estudios reseñados $(18-20,37,38)$.

Aunque este informe es el de un estudio piloto, y el tamaño de la muestra es forzosamente escaso, por lo que las estimaciones de las medias no tienen la precisión de otro estudio de mayor tamaño muestral, podemos concluir que la prevalencia de sintomatología respiratoria en la población rural gallega parece ser elevada, con mayor incidencia en el sexo femenino. La prevalencia de tabaquismo es nuestra población es similar a otras comunidades de nuestro país. Los volúmenes y flujos pulmonares son superiores en el sexo masculino, en los individuos con menos síntomas respiratorios, incluida disnea y en los no fumadores activos. 


\section{Bibliografía}

1. Zervanos NF, Shute KM. Acute, disruptive cough. Symptomatic therapy for a nagging problem. Posgrad Med 1994; 95: 153-64.

2. Sobradillo V, Miravitlles M, Jiménez CA, et al. Estudio IBERPOC en España: prevalencia de síntomas respiratorios habituales y de limitación crónica al flujo aéreo. Arch Bronconeumol 1999; 35 (4): 159-66.

3. González Quijada S, Galdós Barroso M, Riego Valledor A, et al. Neumonía en el anciano: ¿es atípica su presentación clínica?. An Med Interna (Madrid) 2001; 18: 124-126.

4. García Ordóñez MA, Alvarez Hurtado F, Cebrián Gallardo JJ, et al. Neumonía bacteriémica de origen comunitario en el anciano. An Med Interna (Madrid) 1999; 16: 345-348.

5. León Fábregas M, de Diego Damiá A, Perpiñá Tordera M. Utilidad del cuestionario de síntomas respiratorios IUATLD para el diagnóstico diferencial del asma bronquial y la bronquitis crónica. Arch Bronconeumol 2000; 36(8): 441-49.

6. Análise da mortalidade en Galicia 1990. Documentos Técnicos de Saúde Pública. Serie B. Número 3. Dirección Xeral de Saúde Pública. Consellería de Sanidade. Xunta de Galicia.

7. El Futuro de la Neumología en España. Tendencias para el período 1993-2010. Libro Blanco. Estudio Prospectivo Delphi. Sociedad Española de Neumología y Cirugía Torácica (SEPAR). 1993.

8. Becklake M, Crapo RD, Buist S, et al. Lung Function Testing: Selection of reference values and interpretative strategies. Am Rev Respir Dis 1991; 144: 1202-18.

9. Sanchís J y grupo de trabajo de la SEPAR para la práctica de la espirometría en clínica. Arch Bronconeumol 1989; 25: 132-42.

10. Hilleman DE, Dewan N, Malesker M, et al. Pharmacoeconomic evaluation of COPD. Chest 2000; 118: 1278-85.

11. Miravitlles M, Murio C, Guerrero T, et al. Arch Bronconeumol 1999; 35(4): 173-78

12. Giner J, Casan P, Berrojalbiz MA, et al.Cumplimiento de las recomendaciones SEPAR sobre la espirometría forzada. Arch Bronconeumol 1996; 32 (10): 516-22.

13. Miravitlles M, Fernández I, Guerrero T, et al. Desarrollo y resultados de un programa de cribado de la EPOC en atención primaria. El Proyecto PADOC. Arch Bronconeumol 2000; 36 (9): 500-5.

14. Elías Hernández MT, Ortega Ruiz F, Sánchez Riera H, et al. Papel de la disnea en la calidad de vida del paciente con enfermedad pulmonar obstructiva crónica. Arch Bronconeumol 1999; 35(6): 261-66.

15. Rodríguez Portal JA, Alvarez Gutierrez FJ, Segado Soriano A, et al. Arch Bronconeumol 1995; 31:162-68.

16. Antó JM y grupo español del Estudio Europeo del Asma. Prevalencia de síntomas relacionados con el asma en cinco áreas españolas. Med Clin 1995; 104: 487-92.

17. Subirats Bayego A, Vila Ballester Ll, Vila Subirana T, et al. Prevalencia de enfermedades respiratorias en una población rural del norte de Cataluña: La Cerdanya. Med Clin 1994; 103: 481-84.

18. Brotóns B, Pérez JA, Sánchez-Toril F, et al. Prevalencia de la enfermedad pulmonar obstructiva crónica y del asma. Estudio transversal. Arch Bronconeumol 1994; 30: 149-52.

19. Marco Jordán L, Zubillaga Garmendía G, Tapiz Ibáñez V, et al. Encuesta respiratoria en una población rural. Arch Bronconeumol 1992; 28: 165-71.
20. Marco Jordán L, Martín Berra JC, Corres Iñigo M, et al. Enfermedad pulmonar obstructiva crónica en la población general. Estudio epidemiológico realizado en Guipúzcoa. Arch Bronconeumol 1998; 34: 2327.

21. Griffith KA, Sherrill DL, Siegel EM, et al. Predictors of loss of Lung Function in the Elderly. The Cardiovascular Health Study. Am J Respir Crit Care Med 2001; 163: 61-68.

22. Rosenthal M, Bain SH, Cramer D, et al. Lung function in white chidren aged 4 to 19 years: I-Spirometry. Thorax 1993; 48: 794-802.

23. Schrader PC, Quanjer PH, Oliever ICW, et al. Respiratory muscle force and ventilatory function in adolescents. Eur Respir J 1988; 1: 368-75.

24. Burrows B, Cline MG, Knudson RJ, et al. A descriptive analysis of the growth and decline of the FVC and FEV1. Chest 1983; 83 (5): 717-24.

25. Pistelli R, Brancato G, Forastiere F, et al. Population values of lung volumes and flows in children: effect of sex, body mass and respiratory conditions. Eur Respir J 1992; 5: 463-70.

26. Cotes JE, Dabbs JM, Hall Am, et al. Sitting height, fat free mass and body fat as reference variables for lung function in healthy British children; comparison with stature. Ann Hum Biol 1979; 6: 307-14.

27. Leeder SR, Swan AV, Peat JK, et al. Maximum expiratory flow-volume curves in children: changes with growth and individual variability. Bull Europ Physiopath Resp 1977; 13: 249-60.

28. Simon G, Reid L, Tanner JM, et al. Growth of radiologically determined heart diameter, lung width, and lung health from 5-19 years, with standards for clinical use. Arch Dis Child 1972; 47: 373-81.

29. Schrader Pc, Quanjer PH, Borsboom G, et al. Evaluating lung function and anthropometric growth data in a longitudinal study on adolescents. Hum Biol 1984; 56: 365-81.

30. Schwartz JD, Katz SA, Fegley RW, et al. Analysis of spirometric data from a national sample of healthy 6- to 24-year-olds (NHANES II). Am Rev Respir Dis 1988; 138: 1405-14.

31. Schwartz JD, Katz SA, Fegley RW, et al. Sex and race differences in the development of lung function. Am Rev Respir Dis 1988; 138: 1415-21.

32. Zapletal A, Samanek M, Tuma S, et al. Assesment of airway function in children. Etude fonctionelle des voies aeriennes chez l'enfant. Bull Eur Physiopahol Respir 1972; 8: 535-43.

33. Thurlbeck WM. Postnatal human lung growth. Thorax 1982; 37: 564 71.

34. Tanner JM, Whitehouse RH, Marubini et al. The adolescent growth spurt of boys and girls of the Harpender Growth Study. Ann Hum Biol 1976; 3: 109-26.

35. Polgar G, Weng TR. The functional development of the respiratory system. From the period of gestation to adulthood. Am Rev Respir Dis 1979; 120: 625-95.

36. Sharp DS, Enright PL, Chiu D, et al. Reference values for pulmonary function tests of Japanese-American men aged 71 to 90 years. Am J Respir Crit Care Med 1996; 153: 805-11.

37. Zamarrón C, Gude F, Otero Y, et al. Prevalence of sleep disordered breathing and sleep apnea in 50- to 70-year-old individuals. Respiration 1999; 66: 317-22.

38. Gore CJ, Crockett AJ, Pederson DG, et al. Spirometric standards for healthy adult lifetime nonsmokers in Australia. Eur Respir J 1995; 8: 773-82. 